\title{
High expression of spermatogenesis associated serine rich 2 promotes tumorigenicity in esophageal squamous cell carcinoma cells and is associated with poor patient prognosis
}

\author{
YAN-PENG LIU ${ }^{1}$, QIUHONG CAO ${ }^{2},{\text { LING } \mathrm{LI}^{3} \text { and MIN ZHANG }}^{1}$ \\ ${ }^{1}$ Department of Internal Medicine, The Second Hospital of Shandong University, Jinan, Shandong 250033; \\ ${ }^{2}$ Department of Anesthesiology, Jinan Central Hospital, Jinan, Shandong 250013; ${ }^{3}$ Department of Thoracic Surgery, \\ Affiliated Hospital of Shandong Academy of Medical Sciences, Jinan, Shandong 250031, P.R. China
}

Received February 18, 2019; Accepted February 26, 2020

DOI: $10.3892 / \mathrm{etm} .2021 .10130$

\begin{abstract}
Spermatogenesis associated serine rich 2 (SPATS2), recognized as a cytoplasmic RNA-binding protein, is implicated in the tumorgenicity of several cancers. However, the potential role of SPATS2 in esophageal squamous cell carcinoma (ESCC) is yet to be elucidated. The present study aimed to explore the functional implication of SPATS2 in ESCC. The ESCC cell lines Eca109 and KYSE-150 were used to conduct loss-of-function experiments. The expression patterns of SPATS2 in patients with ESCC were obtained from Oncomine, The Cancer Genome Atlas and Genotype-Tissue Expression databases. Reverse transcription-quantitative PCR and western blot analysis were applied to determine the expression levels of SPATS2 in ESCC cells. The proliferation of ESCC cells was measured via cell proliferation and colony-formation assays. Subsequently, the migration and invasion capacities of ESCC cells were observed using Transwell assays. Finally, the expression levels of P53, cyclin E, matrix metalloproteinase (MMP)-9 and neuronal-cadherin were determined via western blot analysis. SPATS2 was expressed at higher levels in ESCC tissues compared with the controls, and high expression of SPATS2 was associated with poor prognosis. ESCC cell line proliferation, migration and invasion abilities were suppressed after silencing SPATS2. Moreover, following knockdown of SPATS2, the proteins cyclin E, MMP-9 and N-cadherin were expressed at markedly decreased levels, while P53 expression was increased. In summary, the results of the present study suggest that SPATS2 promotes ESCC development and progression, providing potential insights into future ESCC targeted treatment.
\end{abstract}

Correspondence to: Dr Min Zhang, Department of Internal Medicine, The Second Hospital of Shandong University, 247 Bei Yuan Street, Jinan, Shandong 250033, P.R. China

E-mail: zhangmin_jinan@163.com

Key words: spermatogenesis associated serine rich 2, esophageal squamous cell carcinoma, proliferation, invasion, migration

\section{Introduction}

Esophageal cancer, one of the most common malignancies worldwide, can be stratified according to its pathology into two subtypes: Esophageal squamous cell carcinoma (ESCC) and esophageal adenocarcinoma $(1,2)$. At present, the majority of esophageal cancers take the form of ESCC (3). The early clinical symptoms of ESCC are not obvious, while at the late stages the disease is associated with lymphatic and hematogenous metastasis (4). In recent years, progress has been made in the screening, diagnosis and treatment of ESCC; however, its incidence is still very high and the prognosis remains not ideal, accompanied by a lower survival rate (5). Due to the lack of biomarkers for early detection and prognosis (6), the 5 -year survival rate is $<10 \%$ after treatment with surgery and chemoradiotherapy (7). Therefore, it is important to identify accurate clinical diagnosis and prognostic markers for ESCC, and to establish new targets for the treatment of these tumors.

The spermatogenesis associated serine rich 2 (SPATS2) gene product is a predicted cytoplasmic RNA binding protein that has been reported to serve a tumorigenic role in several types of tumors, such as colorectal cancer and prostate cancer $(8,9)$. A previous study found that SPATS2 was significantly upregulated in colorectal cancer and promoted cancer cell survival by targeting small nucleolar RNA host gene 5 (9). Ngollo et al (10) reported that SPATS2 was enriched and interacted with $\mathrm{H} 3 \mathrm{~K} 27 \mathrm{me} 3$ to promote prostate cancer aggressiveness. In lung cancer, Takamochi et al (11) discovered that SPATS2 acted as a marker to discriminate squamous cell carcinoma and adenocarcinoma. SPATS2L, an important paralog of SPATS2, is potentially associated with ribosomal processes and translational control (12), and ribosomal biogenesis has been demonstrated to regulate cell growth and proliferation (13). Meanwhile, SPATS2L was also established to serve a role in the occurrence and progression of hepatocellular carcinoma, in combination with microRNA-1269a (14). However, the function of SPATS2 in ESCC remains largely unclear.

The present study aimed to investigate the relationship between the expression of SPATS2 and the prognosis of patients with ESCC. In addition, the regulation of SPATS2 on 
the proliferation, apoptosis, invasion and migration of ESCC cells was also explored in this study.

\section{Materials and methods}

Data acquisition. Expression patterns of SPATS2 in ESCC tissues were assessed using data obtained from the Oncomine database (version 4.5, https://www.oncomine.org; dataset GSE20347) including 17 esophageal and 17 ESCC tissues. Additional data from The Cancer Genome Atlas (TCGA; https://cancergenome.nih.gov/) database containing $182 \mathrm{ESCC}$ tissues and 13 normal tissues (adjacent to the tumor) were used to confirm the expression of SPATS2 in ESCC. The expression of SPATS2 in ESCC was further compared in an analysis using tumor tissue data from the TCGA database $(n=182)$ and normal tissues from the TCGA database $(n=13)$ and samples from the Genotype-Tissue Expression (GTEx) database $(n=273)$. The data from the TCGA and GTEx databases was processed using the Gene Expression Profiling Interactive Analysis (GEPIA1; http://gepia.cancer-pku.cn/) website. Prognostic analysis directed at SPATS2 in patients with ESCC was acquired from the GEPIA website.

Cell culture. Cell lines, including the ESCC cell lines Eca109, KYSE-150 and TE-1, and normal control cell line human esophageal epithelial cells (HEEC) were purchased from The Cell Bank of Type Culture Collection of the Chinese Academy of Sciences. Cells were incubated in RPMI-1640 (Gibco; Thermo Fisher Scientific, Inc.) culture solution with $10 \%$ fetal bovine serum (FBS, Gibco; Thermo Fisher Scientific, Inc.), $100 \mathrm{U} / \mathrm{ml}$ penicillin (Shanghai Qiaoxing Trading Co., Ltd.; http://www.bridge-star.com/qiaoxing2012-Products-18067360/) and $0.1 \mathrm{mg} / \mathrm{ml}$ streptomycin (Shanghai Qiaoxing Trading Co., Ltd, China) at $37^{\circ} \mathrm{C}$ and $5 \% \mathrm{CO}_{2}$.

Cell transfection. Lipofectamine 2000 transfection reagent (Thermo Fisher Scientific, Inc.) was used to transfect Eca109 and KY-SE150 cells in the logarithmic growth stage, according to the manufacturer's guidelines. After $24 \mathrm{~h}$, transfection efficiency was determined and subsequent experiments were performed. The sequences of small interfering si-SPATS2 (20 nM) and si-control (con; $25 \mathrm{nM}$ ) were synthesized by Shanghai GenePharma Co., Ltd., and the primer sequences were as follows: si-SPATS2\#1: 5'-GCACTTTGTTAGTGA ACGTAA-3'; si-SPATS2\#2: 5'-CCCGATGTAGCTCAGTTA CAT-3' and si-con: 5'-AATTCTCCGAACGGTCACGT-3'.

Reverse transcription-quantitative polymerase chain reaction $(R T-q P C R)$. Total RNA was extracted from Eca109 and KY-SE150 cells using TRIzol ${ }^{\circledR}$ reagent (Invitrogen; Thermo Fisher Scientific, Inc.) and then reverse transcribed into cDNA using SuperScript III reverse transcriptase (Invitrogen; Thermo Fisher Scientific, Inc.) according to the manufacturer's protocol. The thermocycling conditions of the RT reaction was as follows: Initial annealing at $37^{\circ} \mathrm{C}$ for $8 \mathrm{~min}$, extension at $42^{\circ} \mathrm{C}$ for $1 \mathrm{~h}$ and termination at $85^{\circ} \mathrm{C}$ for $2 \mathrm{~min}$. SYBR Green Master Mix (Applied Biosystems; Thermo Fisher Scientific, Inc.) was used to determine the relative expression of mRNA. The thermocycling procedure was incubation at $95^{\circ} \mathrm{C}$ for $5 \mathrm{~min}$, followed by 40 cycles of $95^{\circ} \mathrm{C}$ for $30 \mathrm{sec}$ and $60^{\circ} \mathrm{C}$ for
$45 \mathrm{sec}$ before a further $72^{\circ} \mathrm{C}$ incubation for $30 \mathrm{~min}$. GAPDH was used as an internal control to evaluate mRNA expression. The relative expression of mRNA was analyzed using the $2^{-\Delta \Delta \mathrm{Cq}}$ method (15) and the primer sequences were as follows: SPATS2 forward, 5'-CTTTGTCCCCAACCCTCTCC-3' and reverse, 5'-GATCCTCCACCTCCCCTTCT-3'; and GAPDH forward, 5'-GCTCTCTGCTCCTCCTGTTC-3' and, reverse: 5'-AAGTGGTCGTTGAGGGCAATG-3'.

Western blot analysis. Protein was extracted from treated cells including Eca109 and KY-SE150 using RIPA buffer (Beijing Solarbio Science \& Technology Co., Ltd.) with protease inhibitor (PMSF; Abcam) and quantified using a bicinchoninic acid protein assay kit (Thermo Fisher Scientific, Inc.). Equal amounts of protein $(20 \mu \mathrm{g})$ were separated using 12\% SDS-PAGE, and then transferred onto PVDF membranes. Subsequently, the membranes were incubated in 5\% skimmed milk for $1 \mathrm{~h}$ at room temperature and then incubated with primary antibodies (Abcam), including SPATS2 (cat. no. ab122495; 1:500), cyclin E (cat. no. ab33911; 1:1,000), P53 (cat. no. ab32389; 1:1,000), matrix metalloproteinase (MMP-9; cat. no. ab73734; $1 \mu \mathrm{g} / \mathrm{ml}$ ), N-cadherin (cat. no. ab202030; 1:500), and GAPDH (cat. no. ab181602; 1:10,000) at $4^{\circ} \mathrm{C}$ overnight. After washing the membranes with TBS-Tween-20 (0.1\%) three times, they were incubated with secondary antibody goat anti-rabbit IgG H\&L (cat. no. ab205718; 1:2,000; Abcam) at room temperature for $1 \mathrm{~h}$. After washing the membranes three times with TBS-T, the signals were detected using enhanced chemiluminescent reagent (Beyotime Institute of Biotechnology) and densitometry was estimated using Quantity One software (v4.6.6; Bio-Rad Laboratories, Inc.). GAPDH was used as a normalization control.

Cell proliferation assays. Cell Counting Kit-8 (CCK-8) reagent (Dojindo Molecular Technologies, Inc.) was used to assess the viability of Eca109 and KY-SE150 cells according to the manufacturer's protocol. Cells in the logarithmic phase were transfected with si-SPATS2 for $24 \mathrm{~h}$, and then cultured in 96-well plates at a density of $1 \times 10^{3}$ cells/well under the aforementioned culture conditions $\left(37^{\circ} \mathrm{C}\right.$ with $\left.5 \% \mathrm{CO}_{2}\right)$. A total of $10 \mu \mathrm{l}$ of CCK-8 reagent was added into each well and the cells were cultured for $1.5 \mathrm{~h}$ at $37^{\circ} \mathrm{C}$. The optical density values were measured at $0,24,48$ and $72 \mathrm{~h}$ at a wavelength of $450 \mathrm{~nm}$ using a microplate reader.

Colony-formation assays. Cell colony-formation capabilities were estimated in the logarithmic phase. Cells including Eca109 and KY-SE150 were inoculated into a $60 \mathrm{~mm}$ culture dish at a density of 400 cells/dish and incubated at $37^{\circ} \mathrm{C}$ and $5 \% \mathrm{CO}_{2}$ for $1-2$ weeks. When visible clones appeared, the culture was terminated. Colonies were fixed with $4 \%$ paraformaldehyde for $20 \mathrm{~min}$ and stained with $0.1 \%$ crystal violet for $30 \mathrm{~min}$ at room temperature. At least three images of the colonies were captured under a light microscope (magnification, $\mathrm{x} 400)$ and counted.

Transwell assay. The invasion and migration abilities of Eca109 and KY-SE150 cells were determined using Transwell chambers (Corning, Inc.) with or without Matrigel (BD Biosciences). For cell invasion assays, the chambers were pre-coated with Matrigel. Coating was performed by adding 

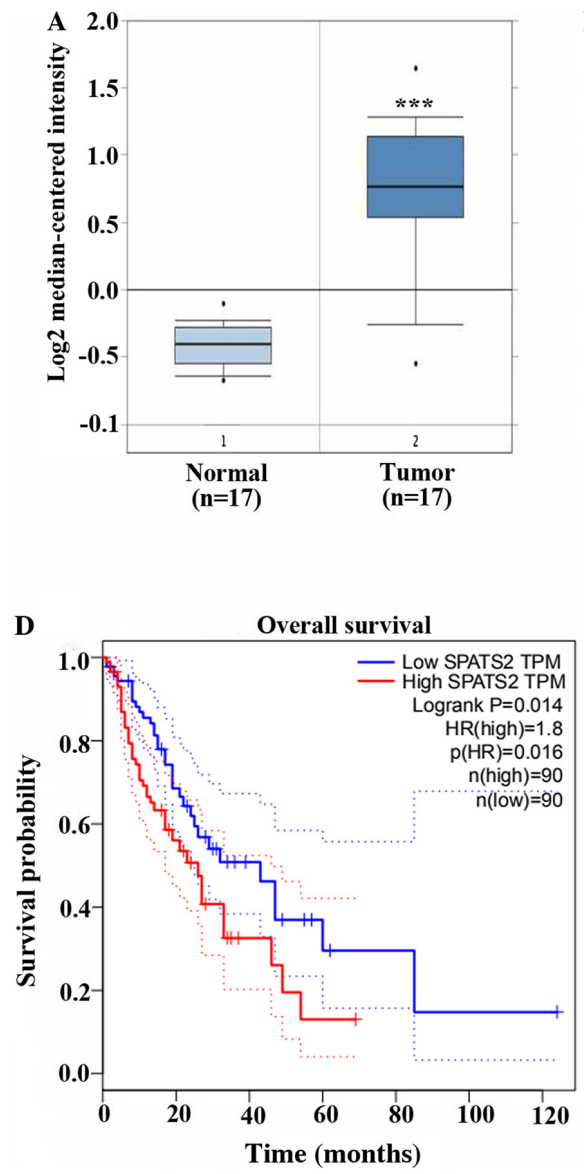
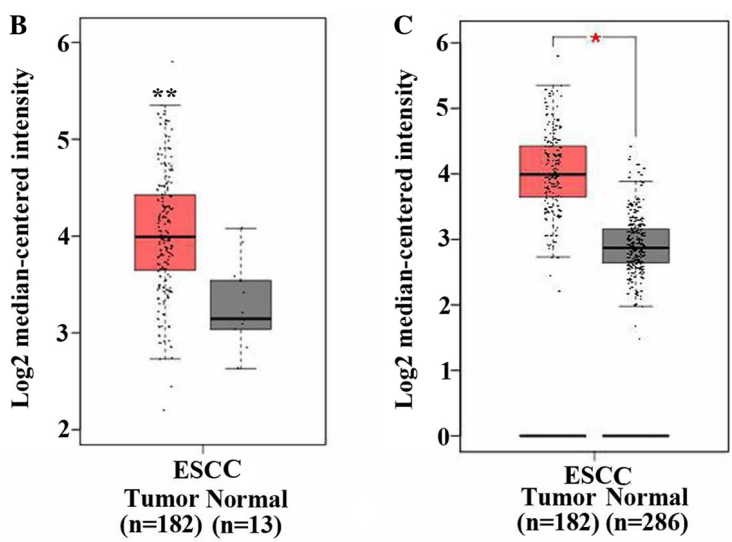

$\mathbf{E}$

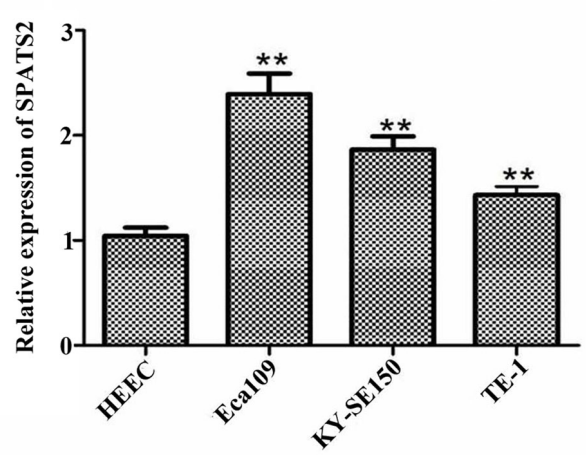

Figure 1. SPATS2 is upregulated in ESCC samples and is associated with a poor prognosis in patients with ESCC. (A) Data from Oncomine revealed that SPATS2 was upregulated in ESCC tissues compared with normal tissues $(\mathrm{n}=17),{ }^{* * *} \mathrm{P}<0.001$ vs. normal tissues. (B) Data from TCGA including 182 tumor tissues and 13 normal tissues indicated that SPATS2 is upregulated in ESCC tissues ${ }^{* *} \mathrm{P}<0.01$ vs. normal tissues. (C) Data from TCGA (182 tumor tissues and 13 normal tissues) and Gene and Tissue Expression database (273 normal tissues) revealed that SPATS2 was upregulated in tumors compared with the normal tissues, ${ }^{*} \mathrm{P}<0.05$ vs. normal tissues. (D) Patients with high expression of SPATS2 had a less favorable overall survival time, analyzed by gene expression profiling interactive analysis. $\mathrm{P}=0.014$. (E) Expression of SPATS2 was significantly increased in ESCC cell lines compared with normal cell line, ${ }^{* *} \mathrm{P}<0.01 \mathrm{vs}$. HEEC. ESCC, esophageal squamous cell carcinoma; HEEC, human esophageal epithelial cells; SPATS2, spermatogenesis-associated serine rich 2; TCGA, The Cancer Genome Atlas.

$100 \mu 1$ Matrigel into the upper chamber of the Transwell chamber of the 24-well plate, shaken evenly and placed in a $\mathrm{CO}_{2}$ incubator at $37^{\circ} \mathrm{C}$ for $4-6 \mathrm{~h}$ to form a gel. Cell migration assays did not require the chambers to be pre-coated. A total of $100 \mu \mathrm{l}$ cell suspension (containing $5 \times 10^{3}$ cells, excluding serum) was added into the upper chamber and $500 \mu 1$ complete RPMI-1640 medium was added into the lower chamber. After incubation at $37^{\circ} \mathrm{C}$ for $24 \mathrm{~h}$ and removal of the residual cells on the surface of upper chamber, the invasive and migratory cells were fixed with $4 \%$ paraformaldehyde for $30 \mathrm{~min}$ and stained with $0.1 \%$ crystal violet for $20 \mathrm{~min}$ at room temperature. A total of 5 fields were randomly selected and images captured by a light microscope (magnification, $\mathrm{x} 400$ ).

Statistical analysis. All experiments were repeated at least three times. Experimental data was analyzed using SPSS version 22.0 (IBM Corp.) and GraphPad Prism version 5.0 (GraphPad Software, Inc.). Unpaired Student's t-test was performed to analyze the difference between two groups and one-way ANOVA analysis with Dunnett's post hoc test was performed for comparison among multiple samples. Survival curves were analyzed by the Kaplan-Meier method and log-rank test. In survival analysis,
ESCC patients were divided into a high expression group and low expression group according to SPATS2 expression level. The median expression level values were used to define high and low expression of SPATS2 in ESCC, the median value in tumor was calculated to be 14.92 whereas in that the normal group is 6.31. All experiments were independently repeated three times. ${ }^{*} \mathrm{P}<0.05$ was considered statistically significant.

\section{Results}

High expression of SPATS2 is associated with a less favorable overall survival time in patients with ESCC. In order to explore the function of SPATS2 in ESCC, SPATS2 expression patterns in patient samples were derived from a number of public databases. Data acquired from Oncomine indicated that SPATS2 was expressed at higher levels in ESCC tissues $(\mathrm{n}=17)$ compared with normal tissues ( $\mathrm{n}=17$; Fig. $1 \mathrm{~A}$; $\mathrm{P}<0.0001)$. SPATS2 was also upregulated in patients with ESCC compared with normal tissues according to data from TCGA, including 182 tumor tissues and 13 normal tissues (Fig. 1B; P<0.01). A comparison of cancer samples and normal tissue data derived from TCGA including 182 tumor tissues and 13 normal tissues 

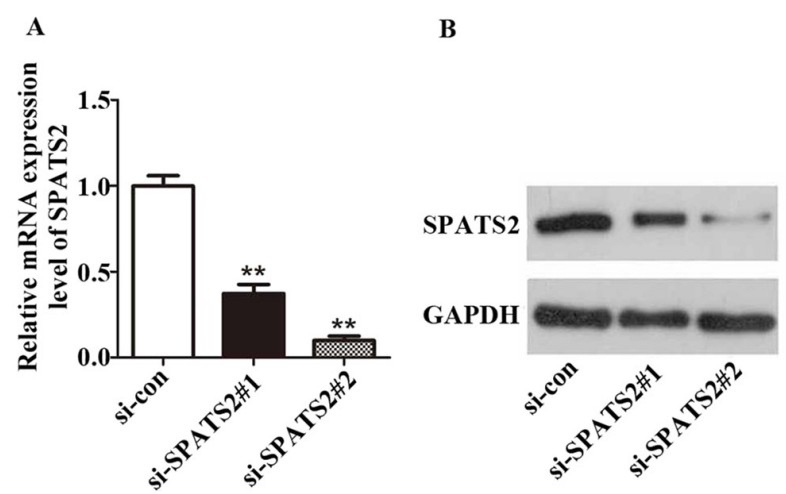

Eca109
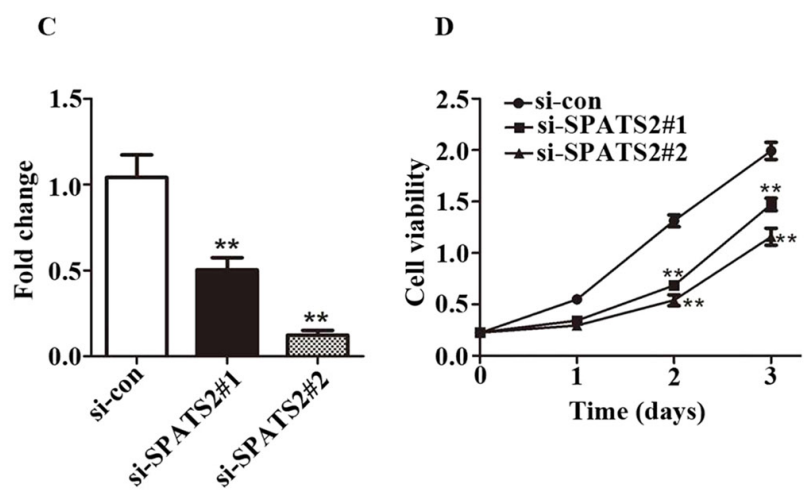

$\mathbf{E}$

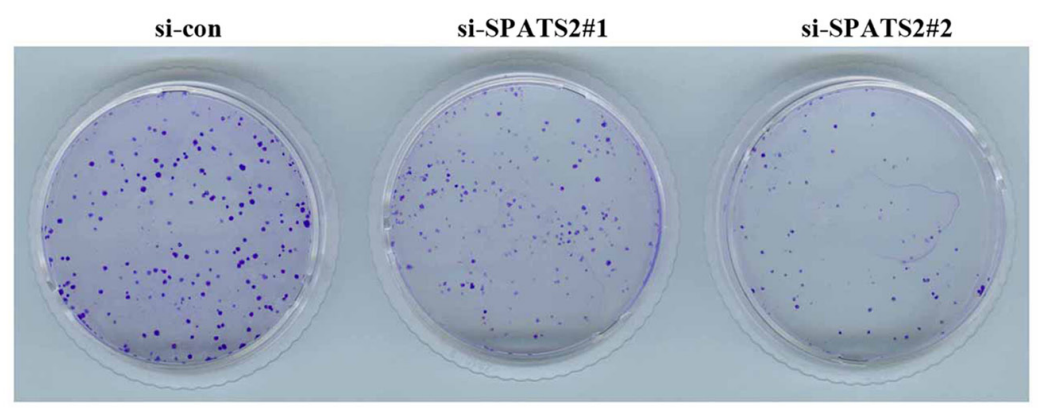

$\mathbf{F}$

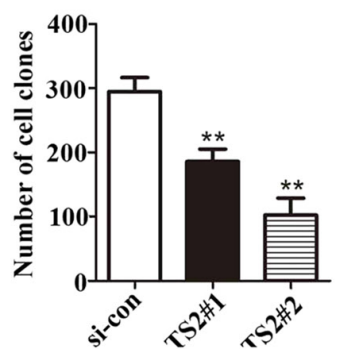

G

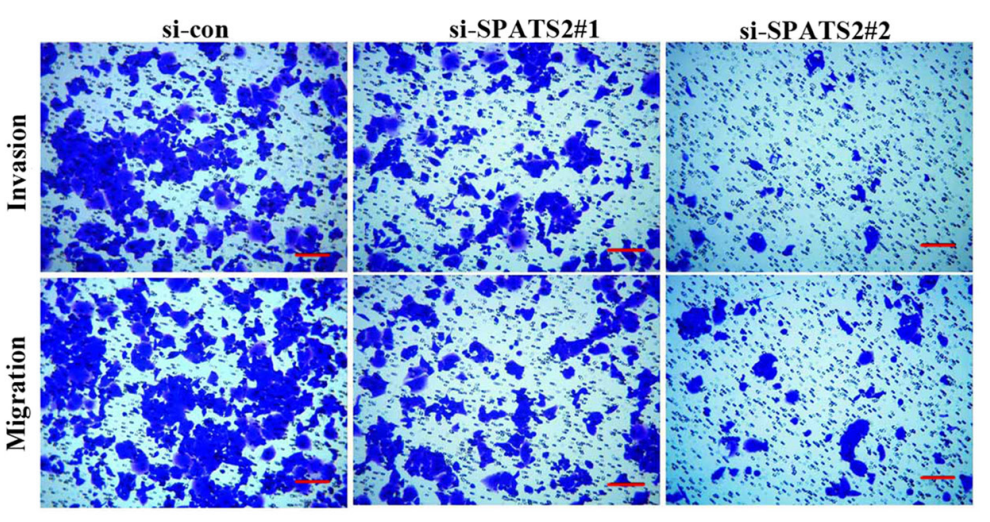

$\mathbf{H}$

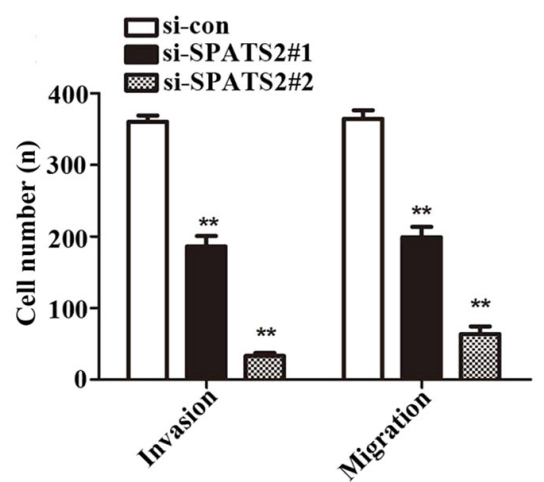

Figure 2. Silencing SPATS2 represses Eca109 cell proliferation, colony formation, migration and invasion capabilities. (A) mRNA levels and (B) representative western blots indicated that SPATS2 expression decreased after cells were transfected with si-SPATS2, compared with control. (C) Western blotting densitometry. (D) Cell proliferation was reduced after transfection with si-SPATS2 compared with control. (E) Representative images of colonies formed. (F) Number of colonies formed was significantly decreast after transfection with si-SPATS2 compared with si-con. ${ }^{* *} \mathrm{P}<0.01$ vs. si-con. con, control; si, small interfering; SPATS2 spermatogenesis-associated serine rich 2. (G) Representative images of migratory and invasive cells. (H) Statistical analysis indicated that migratory and invasive cell numbers declined after knockdown of SPATS2, compared with si-con. Scale bar $=200 \mu \mathrm{m},{ }^{* *} \mathrm{P}<0.01 \mathrm{vs}$. si-con. con, control; si, small interfering; SPATS2 spermatogenesis-associated serine rich 2.

and GTEx containing 273 normal tissues revealed that SPATS2 was upregulated in tumor tissues by contrast to normal tissues (Fig. 1C; $\mathrm{P}<0.05$ ). Moreover, high expression of SPATS2 was associated with a shorter survival time in patients with ESCC (Fig. 1D; $\mathrm{P}=0.014$ ).

Subsequently, the mRNA expression of SPATS2 was investigated in three ESCC cell lines, Eca109, KY-SE150 and TE-1, and the normal cell line HEEC. As indicated in Fig. 1E, the expression of SPATS2 was significantly increased in ESCC cell lines compared with a normal cell line $(\mathrm{P}<0.01)$. These data indicate that SPATS2 was upregulated in ESCC tissues and cell lines and was associated with a poor prognosis in patients with ESCC.
Knockdown of SPATS2 suppresses cell proliferation in ESCC. To further investigate the association between SPATS2 and ESCC progression, two ESCC cell lines, Eca109 and KYSE-150, were examined. Of the ESCC lines investigated, SPATS2 was expressed at the highest levels in these two cell lines (Fig. 1E). siRNA was transfected in order to silence SPATS2 in ESCC cells. As indicated in Figs. 2A-C and 3A-C, the expression of SPATS2 was suppressed at both the mRNA and protein level after transfection with si-SPATS2 when compared with si-con, providing a basis for subsequent experiments $(\mathrm{P}<0.01)$.

A CCK-8 kit was used to determine ESCC cell viability. The results indicated that Eca109 and KYSE-150 cell proliferation 


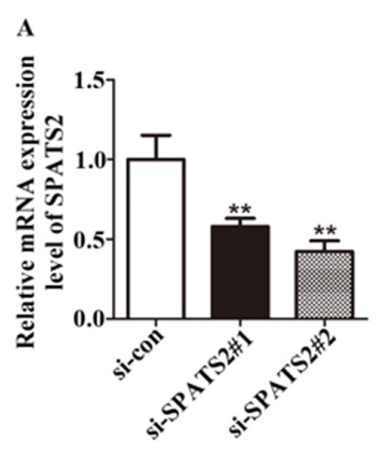

E

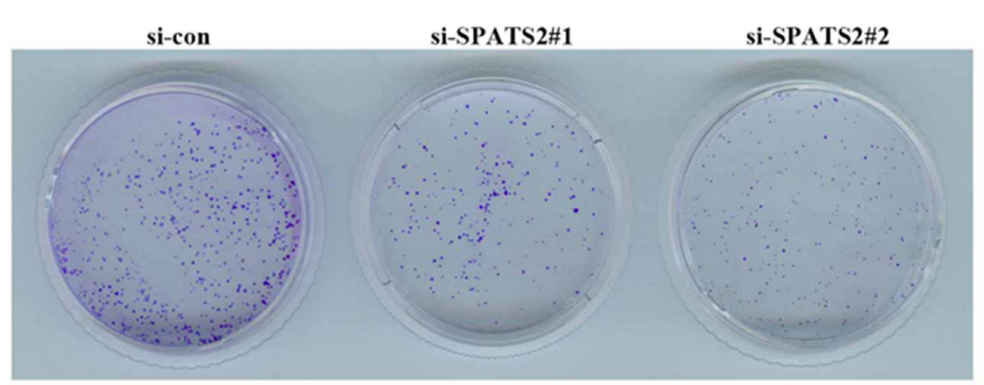

G

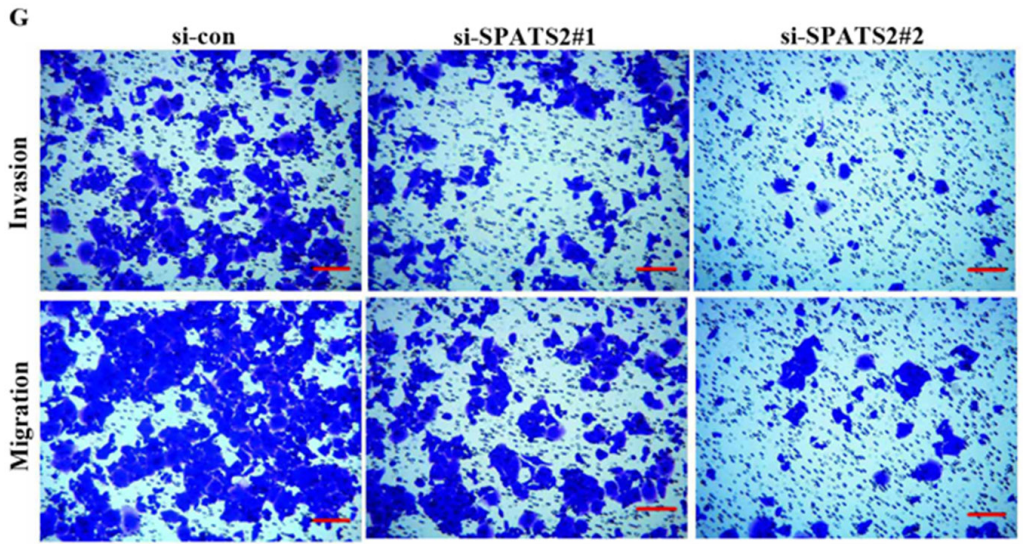

KY-SE150

B

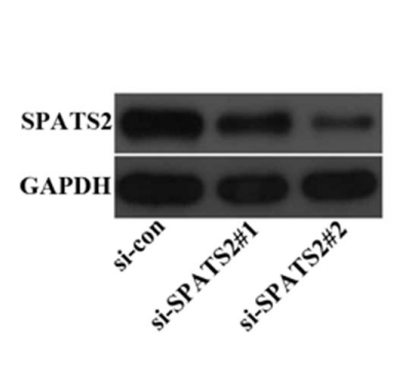

D

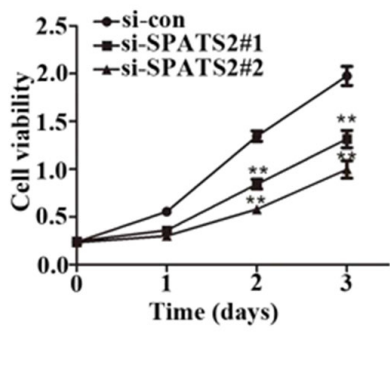

F

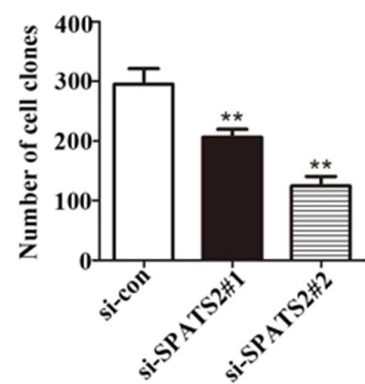

H

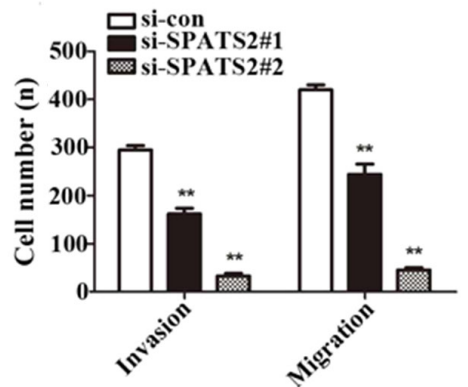

Figure 3. Knockdown of SPATS2 suppresses KY-SE150 cell proliferation, colony formation, invasion and migration abilities. (A) mRNA levels and (B) representative western blots of SPATS2 in KY-SE150 cells after treatment with si-SPATS2 and si-con. (C) Densitometry of western blots. (D) Silencing of SPATS2 inhibited KY-SE150 cell proliferation. (E) Representative images and (F) the number of clones in KY-SE150 cells after siRNA-mediated knockdown of SPATS2 (G) Representative images and (H) numbers of invasive and migratory KY-SE150 cells. Scale bar $=200 \mu \mathrm{m},{ }^{* * *} \mathrm{P}<0.01 \mathrm{vs}$. si-con. con, control; si, small interfering; SPATS2 spermatogenesis-associated serine rich 2.

A

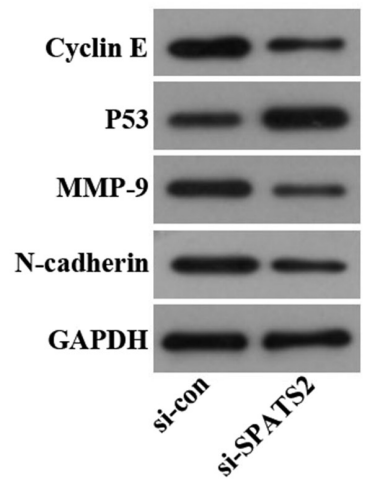

B

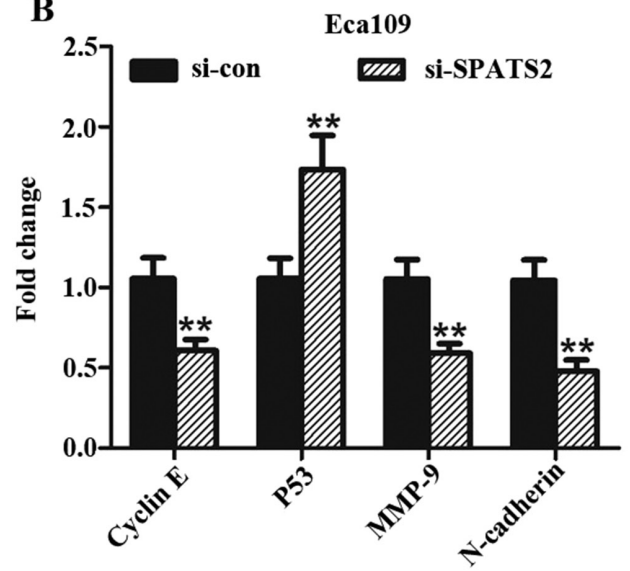

Figure 4. SPATS2 is associated with key proteins associated with esophageal squamous cell carcinoma progression. (A) Representative western blots of cyclin E, MMP-9, N-cadherin and P53 in Eca109 cells were (B) quantified after knockdown of SPATS2 using si-SPATS2\#2. ${ }^{* *}$ P<0.01 vs. si-con. con, control; MMP, matrix metalloproteinase; si, small interfering; SPATS2 spermatogenesis-associated serine rich 2. 
was significantly inhibited at 48 and $72 \mathrm{~h}$ after knockdown of SPATS2 when compared with the control (Figs. 2D and 3D; $\mathrm{P}<0.01)$.

According to the observed link between cell viability and SPATS2 expression, a colony formation assay was designed to assess the influence of SPATS2 on ESCC cells. The results indicated that colony formation ability was significantly decreased after knockdown of SPATS2, compared with the control (Figs. 2E-F and 3E-F; P<0.01). Taken together, the current findings indicate that downregulation of SPATS2 repressed the proliferation of ESCC cells.

SPATS2 serves an active role in migration and invasion of ESCC cells. Next, a Transwell assay was used to explore the migration and invasion abilities of ESCC cells, after silencing of SPATS2. As revealed in Figs. 2G-H, and 3G-H, migratory and invasive cells numbers declined after knockdown of SPATS2 in contrast with the si-con group $(\mathrm{P}<0.01)$. The results suggested that downregulation of SPATS2 suppressed the migratory and invasive capacity of ESCC cells.

Silencing SPATS2 has a significant effect on key proteins in ESCC progression. In the present study, Eca109 cells were used to assess changes in the expression of proteins associated with ESCC progression after knockdown of SPATS2 with si-SPATS2\#2. Following knockdown, the expression levels of cyclin E, MMP-9 and N-cadherin were significantly decreased compared to cells treated with si-con group, while P53 was significantly upregulated (Fig. $4 \mathrm{~A}$ and $\mathrm{B}, \mathrm{P}<0.01$ ). Taken together, the data suggest that SPATS2 may influence ESCC cell proliferation, invasion and migration abilities, partly via regulating expression of cyclin E, MMP-9, N-cadherin and P53.

\section{Discussion}

In the present study, SPATS2 was determined to be upregulated in ESCC tissues and cells, and this was significantly associated with a less favorable prognosis in patients with ESCC. Subsequently, RNA interference experiments revealed that knockdown of SPATS2 inhibited ESCC cell proliferation, migration and invasion. In addition, knockdown of SPATS2 influenced the expression of key proteins associated with the progression of ESCC. Taken together, the present data indicate that SPATS2 may facilitate tumorigenesis in ESCC via its influence on cell proliferation, migration and invasion.

ESCC has inconspicuous early symptoms, high incidence and poor prognosis, and for these reasons developing new approaches to treatment is important (16). Numerous possible biomarkers of ESCC have been reported, but none have been widely applied to guide clinical studies (6). As SPATS2 is a relatively recently discovered gene, limited research into its potential roles has been performed (8). The earliest study into SPATS2 reported that its expression was induced by bisphenol A downregulation in oral epithelial cells (17). Recently, increased expression of SPATS2 was found both in colorectal and prostate cancer $(9,10)$. As the upstream interaction protein of SPATS2, neuroblastoma RAS viral oncogene homolog (NRAS) plays an important role in the occurrence and metastasis of various cancers, including colorectal (18), primary thyroid (19), lung (20) and bladder cancer (21). In the present study, SPATS2 was revealed to be highly expressed in ESCC tissues and cells, and silencing of SPATS2 had negative effects on cell proliferation, invasion and migration. The present data together with previous reports indicates that SPATS2 may serve an active role in the occurrence and progression of ESCC.

Alterations in the mechanism controlling apoptosis and cell cycle progression influence the pathogenesis of different human neoplasia (22). P53, as a tumor suppressor gene, is influences numerous types of cancer, including ESCC (23). P53 dysfunction is frequently observed in tumors and regulates molecular mechanisms underlying tumor progression (24). Cyclin E is a key regulatory factor of the cell cycle (25), promoting DNA duplication and centrosome replication in the late $G_{1}$ stage, and is highly expressed in cancers of the digestive system (26). MMPs are important contributors to metastasis and have been revealed to promote cell invasion in numerous human cancers (27). MMP-9 is a protease, which is upregulated in the majority of malignancies (28), including ESCC, and has been demonstrated to enhance cell invasion and metastasis (29). $\mathrm{N}$-cadherin promotes the invasion of cancer cells and is highly expressed in a range cancer types. Expression of N-cadherin in epithelial cells causes changes in morphology to a fibroblastic phenotype, causing the cells to become more motile and invasive (30). In addition, $\mathrm{N}$-cadherin has been reported to promote cell adhesion and regulate tumor progression (31). All of these genes are crucial to the development of tumors and are regarded as makers for tumor progression. Therefore, in the present study, in order to explore the molecular mechanism of ESCC following SPATS2 depletion, P53, MMP-9, cyclin $\mathrm{E}$ and $\mathrm{N}$-cadherin levels were assessed. The results indicated that protein expression of MMP-9, cyclin E and N-cadherin decrease compared with the control, after silencing of SPATS2. By contrast, P53 was significantly upregulated. The aforementioned results indicate that SPATS2 may affect the proliferation, migration and invasion abilities of ESCC cells via regulating the expression of these proteins. Notably, a limitation of the present research was the lack of in vivo experiments to confirm the current results.

In summary, SPATS2 was highly expressed in ESCC tissues and cells, and its upregulation was significantly associated with a poor prognosis in patients with ESCC. Furthermore, knockdown of SPATS2 suppressed cell proliferation, invasion and migration abilities by regulating key proteins involved in ESCC progression. The current findings suggest that SPATS2 expression may be an important factor in the prognosis of patients with ESCC, providing a new potential target for the treatment of ESCC tumors.

\section{Acknowledgements}

Not applicable.

\section{Funding}

No funding was received.

\section{Availability of data and materials}

The datasets used and analyzed during the current study are available from the corresponding author on reasonable request. 


\section{Authors' contributions}

YPL analyzed the data from biological websites, and performed the biological experiments, and edited the manuscript. QHC performed the biological experiments and edited the manuscript. LL analyzed the experimental data and edited the manuscript. MZ processed the experimental data and revised the manuscript. All authors read and approved the final manuscript.

\section{Ethics approval and consent to participate}

Not applicable.

\section{Patient consent for publication}

Not applicable.

\section{Competing interests}

The authors declare that they have no competing interests.

\section{References}

1. Hu JM, Liu K, Liu JH, Jiang XL, Wang XL, Chen YZ, Li SG, Zou H, Pang LJ, Liu CX, et al: CD163 as a marker of M2 macrophage, contribute to predict aggressiveness and prognosis of Kazakh esophageal squamous cell carcinoma. Oncotarget 8: 21526-21538, 2017.

2. Sakai NS, Samia-Aly E, Barbera M and Fitzgerald RC: A review of the current understanding and clinical utility of miRNAs in esophageal cancer. Semin Cancer Biol 23: 512-521, 2013.

3. Torre LA, Bray F, Siegel RL, Ferlay J, Lortet-Tieulent J and Jemal A: Global cancer statistics, 2012. CA Cancer J Clin 65: 87-108, 2015.

4. Murphy G, McCormack V, Abedi-Ardekani B, Arnold M, Camargo MC, Dar NA, Dawsey SM, Etemadi A, Fitzgerald RC, Fleischer DE, et al: International cancer seminars: A focus on esophageal squamous cell carcinoma. Ann Oncol 28: 2086-2093, 2017.

5. Gao S, Li S, Duan X, Gu Z, Ma Z, Yuan X, Feng X and Wang H: Inhibition of glycogen synthase kinase 3 beta (GSK3beta) suppresses the progression of esophageal squamous cell carcinoma by modifying STAT3 activity. Mol Carcinog 56: 2301-2316, 2017.

6. Wang C, Wang J, Chen Z, Gao Y and He J: Immunohistochemical prognostic markers of esophageal squamous cell carcinoma: A systematic review. Chin J Cancer 36: 65, 2017.

7. Ekman S, Dreilich M, Lennartsson J, Wallner B, Brattstrom D, Sundbom M and Bergqvist M: Esophageal cancer: Current and emerging therapy modalities. Expert Rev Anticancer Ther 8: 1433-1448, 2008.

8. Senoo M, Hoshino S, Mochida N, Matsumura Y and Habu S: Identification of a novel protein p59(scr), which is expressed at specific stages of mouse spermatogenesis. Biochem Biophys Res Commun 292: 992-998, 2002.

9. Damas ND, Marcatti M, Come C, Christensen LL, Nielsen MM, Baumgartner R, Gylling HM, Maglieri G, Rundsten CF, Seemann SE, et al: SNHG5 promotes colorectal cancer cell survival by counteracting STAU1-mediated mRNA destabilization. Nat Commun 7: 13875, 2016

10. Ngollo M, Lebert A, Daures M, Judes G, Rifai K, Dubois L, Kemeny JL, Penault-Llorca F, Bignon YJ, Guy L and Bernard-Gallon D: Global analysis of H3K27me3 as an epigenetic marker in prostate cancer progression. BMC Cancer 17: 261, 2017.

11. Takamochi K, Ohmiya H, Itoh M, Mogushi K, Saito T, Hara K, Mitani K, Kogo Y, Yamanaka Y, Kawai J, et al: Novel biomarkers that assist in accurate discrimination of squamous cell carcinoma from adenocarcinoma of the lung. BMC Cancer 16: 760, 2016

12. Zhu CH, Kim J, Shay JW and Wright WE: SGNP: An essential Stress Granule/Nucleolar Protein potentially involved in $5.8 \mathrm{~s}$ rRNA processing/transport. PLoS One 3: e3716, 2008.

13. Donati G, Montanaro L and Derenzini M: Ribosome biogenesis and control of cell proliferation: p53 is not alone. Cancer Res 72: $1602-1607,2012$
14. Min P, Li W, Zeng D, Ma Y, Xu D, Zheng W, Tang F, Chen J, Shi J, Hu H, et al: A single nucleotide variant in microRNA-1269a promotes the occurrence and process of hepatocellular carcinoma by targeting to oncogenes SPATS2L and LRP6. Bull Cancer 104: 311-320, 2017.

15. Livak KJ and Schmittgen TD: Analysis of relative gene expression data using real-time quantitative PCR and the 2(-Delta Delta $\mathrm{C}(\mathrm{T})$ ) method. Methods 25: 402-8, 2001

16. Baba Y, Saeki H, Nakashima Y, Oki E, Shigaki H, Yoshida N, Watanabe M, Maehara Y and Baba H: Review of chemotherapeutic approaches for operable and inoperable esophageal squamous cell carcinoma. Dis Esophagus 30: 1-7, 2017.

17. Seki K, Koshi R, Sugano N, Masutani S, Yoshinuma N and Ito K: Microarray analysis of bisphenol A-induced changes in gene expression in human oral epithelial cells. Acta Biochim Biophys Sin (Shanghai) 39: 879-884, 2007.

18. Cicenas J, Tamosaitis L, Kvederaviciute K, Tarvydas R, Staniute G, Kalyan K, Meskinyte-Kausiliene E, Stankevicius V and Valius M: KRAS, NRAS and BRAF mutations in colorectal cancer and melanoma. Med Oncol 34: 26, 2017.

19. Melo M, Gaspar da Rocha A, Batista R, Vinagre J, Martins MJ, Costa G, Ribeiro C, Carrilho F, Leite V, Lobo C, et al: TERT, BRAF, and NRAS in primary thyroid cancer and metastatic disease. J Clin Endocrinol Metab 102: 1898-1907, 2017.

20. Giannou AD, Marazioti A, Kanellakis NI, Giopanou I, Lilis I, Zazara DE, Ntaliarda G, Kati D, Armenis V, Giotopoulou GA, et al: NRAS destines tumor cells to the lungs. EMBO Mol Med 9: 672-686, 2017.

21. Li Y, Shan Z, Liu C, Yang D, Wu J, Men C and Xu Y: MicroRNA-294 promotes cellular proliferation and motility through the PI3K/AKT and JAK/STAT pathways by Upregulation of NRAS in bladder cancer. Biochemistry (Mosc) 82: 474-482, 2017.

22. De Amicis F, Perri A, Vizza D, Russo A, Panno ML, Bonofiglio D, Giordano C, Mauro L, Aquila S, Tramontano D and Ando S: Epigallocatechin gallate inhibits growth and epithelial-to-mesenchymal transition in human thyroid carcinoma cell lines. J Cell Physiol 228: 2054-2062, 2013.

23. Bellini MF, Cadamuro AC, Succi M, Proenca MA and Silva AE: Alterations of the TP53 gene in gastric and esophageal carcinogenesis. J Biomed Biotechnol 2012: 891961, 2012.

24. Zhang Y, Zhu C, Sun B, Lv J, Liu Z, Liu S and Li H: Integrated high throughput analysis identifies GSK3 as a crucial determinant of p53-mediated apoptosis in lung cancer cells. Cell Physiol Biochem 42: 1177-1191, 2017

25. Dai L, Liu Y, Liu J, Wen X, Xu Z, Wang Z, Sun H, Tang S, Maguire AR, Quan J, et al: A novel cyclinE/cyclinA-CDK inhibitor targets p27(Kip1) degradation, cell cycle progression and cell survival: Implications in cancer therapy. Cancer Lett 333: 103-112, 2013.

26. Huang L, Ren F, Tang R, Feng Z and Chen G: Prognostic value of expression of Cyclin $\mathrm{E}$ in gastrointestinal cancer: A systematic review and meta-analysis. Technol Cancer Res Treat 15: 12-19, 2016.

27. Thaker PH, Han LY, Kamat AA, Arevalo JM, Takahashi R, Lu C, Jennings NB, Armaiz-Pena G, Bankson JA, Ravoori M, et al: Chronic stress promotes tumor growth and angiogenesis in a mouse model of ovarian carcinoma. Nat Med 12: 939-944, 2006.

28. Grunwald B, Vandooren J, Locatelli E, Fiten P, Opdenakker G, Proost P, Kruger A, Lellouche JP, Israel LL, Shenkman L and Comes Franchini M: Matrix metalloproteinase-9 (MMP-9) as an activator of nanosystems for targeted drug delivery in pancreatic cancer. J Control Release 239: 39-48, 2016.

29. Bai X, Li YY, Zhang HY, Wang F, He HL, Yao JC, Liu L and Li SS: Role of matrix metalloproteinase-9 in transforming growth factor- $\beta 1$-induced epithelial-mesenchymal transition in esophageal squamous cell carcinoma. Onco Targets Ther 10: 2837-2847, 2017.

30. Derycke LD and Bracke ME: N-cadherin in the spotlight of cell-cell adhesion, differentiation, embryogenesis, invasion and signalling. Int J Dev Biol 48: 463-476, 2004.

31. Blaschuk OW: N-cadherin antagonists as oncology therapeutics. Philos Trans R Soc Lond B Biol Sci 370: 20140039, 2015.

This work is licensed under a Creative Commons Attribution-NonCommercial-NoDerivatives 4.0 International (CC BY-NC-ND 4.0) License. 